\title{
ON THE APPLICABILITY OF THE ANALYTIC NETWORK PROCESS TO RURAL TELECOMMUNICATIONS INFRASTRUCTURE TECHNOLOGY SELECTION
}

\author{
Yousef Gasiea* \\ School of Mechanical, Aerospace and Civil Engineering \\ The University of Manchester \\ Manchester, UK \\ E-mail: yousef.gasiea@postgrad.manchester.ac.uk \\ Margaret Emsley \\ School of Mechanical, Aerospace and Civil Engineering \\ The University of Manchester \\ Manchester, UK \\ E-mail: margaret.emsley@manchester.ac.uk \\ Ludmil Mikhailov \\ Manchester Business School \\ The University of Manchester \\ Manchester, UK \\ E-mail: ludi.mikhailov@manchester.ac.uk
}

\begin{abstract}
The decisions involved in rural settings are of complex nature, with some aspects compounded by the presence of intangible criteria. Hence, a suitable approach is needed that can produce effective solutions. This paper describes the applicability of a multi-criteria decision-making method, specifically the Analytic Network Process (ANP), to model the selection of an appropriate telecommunications infrastructure technology, capable of deploying e-services in rural areas of developing countries. It aims to raise awareness among telecommunication planners about the availability of ANP, and to demonstrate its suitability to enhance the selection process. It focuses on the ANP main stages, namely: the structuring of the problem and constructing the model, assessment of the importance of the related factors and finally the synthesis of results. The proposed model is constructed based on concerned experts' views of relevant selection criteria and potential technology alternatives. Its network structure caters for all possible dependencies and interactions among criteria and alternatives.
\end{abstract}

Keywords: Analytic Network Process, Rural Telecommunications, Technology Selection.

\section{Introduction}

Telecommunications technology is evolving rapidly and offers information links between urban and rural areas that can overcome distance barriers and provide e-services to these hardly accessible areas. Recent technological advances in transmission systems like fiber optics, wireless and satellite can now supply services to these locations at affordable prices. However, with different criteria for technology evaluation and various telecommunications infrastructure alternatives available nowadays, the selection process becomes complicated; there is uncertainty and multiple conflicting objectives with sociological, demographical, environmental, political, cultural, economical and technical aspects. This raises the need for some kind of structure or model, based on a suitable Multi Criteria Decision Making (MCDM) method.

Some previous applications of MCDM methods, with particular focus on the use of the Analytic Hierarchy Process (AHP) to rural telecommunications include (Nazem et al., 2001), (Chemane et al., 2005), (Sasidhar and Min, 2005), (Nepal, 2005), (Andrew et al., 2005). While all of these papers provide valuable decision

\footnotetext{
* Corresponding author
} 


\section{Y. Gasiea, M. Emsley \& L. Mikhailov}

models, but, very few studies have considered all criteria relevant to rural telecommunications, as most of them obviously apply no factor interactions. For example, if a model's emphasis is mainly technical, then the economic, social, regulatory and environmental criteria are probably not adequately addressed.

Basically, the AHP is a suitable method when interdependencies between factors do not exist (Shang et al., 2004). Its models do not consider important issues such as interaction among and between decision making levels/clusters as well as dependency among qualitative factors. These are important issues in rural telecommunications decision problems which cannot not be structured hierarchically because they involve many interactions and dependencies requiring a MCDM method that can holistically deal with qualitative and quantitative data, with different conflicting objectives, to arrive at a consensus decision in relation to the choice of a suitable rural telecommunication technology.

In this paper, the ANP is chosen because it can successfully handle the above factors. The ANP has some additional advantages over the AHP and other MCDM methods, such as its holistic approach, in which all the factors and criteria involved are laid out in advance in a network system that allows for dependency. Its power lies in its use of special ratio scales to capture interactions for making accurate predictions and reach better decisions (Saaty, 2005). Moreover, its suitability in offering solutions in a complex multicriteria decision environment, together with the availability of software supporting its functions, further acknowledge its applicability to tackle such a problem. It has also proved to be successful in utilising expert knowledge to tackle several selection problems, e.g. (Lee and Kim, 2000) and (Meade and Presley 2002).

To the best knowledge of the authors, applications of the ANP to the selection of rural telecommunications infrastructure technologies have not been tackled. This paper therefore aimed to fill this gap in the literature to particularly allow for the explicit consideration of dependencies and interactions in the decision making process, and still maintain the acknowledged advantages of the AHP method.

\section{The selection of rural telecommunication infrastructure technology}

There is a need to provide access to the main telecommunications network and expand connectivity to such areas, thus enabling the rollout of the appropriate telecommunication services. However, the choice of appropriate telecommunications infrastructure technology that will provide the required e-services within various constraints is a challenge. Typically, technology selection is based on a mixture of different criteria, one of which is the remoteness of a village. If the village is within $35 \mathrm{~km}$ of the nearest local exchange, telecommunication services can be provided to that village using a one-hop last-mile link. However, if the village is further away, at least two transmission hops must be established (Pipattanasomporn, 2004). Hence, two types of telecommunications infrastructure technologies are needed to provide rural telecommunication services, namely Backbone network (Core) and Access network (Last mile).

The backbone network provides the long-haul signal transmission from the country's main telecommunication centre to the remote access network, i.e. trunking services (Pipattanasomporn, 2004). This network may be wireless or wireline, including analogue and digital transmission technology over Fiber Optic, Wireless or Satellite transmission media (Chemane et al., 2005). The access network provides the connectivity between the end-user and the backbone network and may be based on wireless or wireline technologies, e.g. copper wires or wireless, connected to network nodes at the edge of the backbone network. Technologies in both networks can be circuit-switched or packet-switched. Any decision made for each of these two segments must take into account the characteristics of rural settlements.

The primary focus of this paper is mainly on the backbone network by attempting to provide a structure of the decision problem and proposing a technology selection model of such an infrastructure. The telecommunications backbone is, in general, a key problem for rural information infrastructure, as low population density is linked to high cost of service for any communications technology, especially for wireline services. It poses the greatest challenge to bringing affordable telecommunication services to rural residents. However, once it is in place and running, it will be possible to connect other nearby rural villages with a wide range of telecommunication technologies and needed services.

The infrastructure technology selection process, especially in the case of rural telecommunications in developing countries, is a multi-faceted, multi-criteria decision making problem, requiring consideration of 
some wide-ranging qualitative factors related to socio-economical and political issues. These are hard to quantify and will have great impact on the selection process, in respect of the social, environmental, regulatory and demographical concerns, etc. Furthermore, in order to incorporate other tangible factors, in the absence of past statistical data to analyse, such as technical and economical related factors etc., it is necessary to use a suitable multi-criteria method for analysis and synthesis by a group of experts rather than an individual. A telecommunication operator usually receives several technology solutions from external vendors. The challenge of matching the parameters of an engineering problem to the available solutions becomes a challenge to the telecoms engineer in this particular selection phase (Andrew et al., 2005).

An ANP-based decision model is therefore proposed as a suitable methodology because "decisions obtained from a network can be significantly different from those obtained from a more complex hierarchy" (Saaty, 1996). It is constructed to include an in-depth and comprehensive examination of all pertinent factors and will be dependent on the perceptual weightings, provided by telecommunications experts.

\section{The Analytic Network Process}

The ANP is a multi-attribute decision making approach developed by Thomas L. Saaty and was originally called the supermatrix technique (Saaty, 2001). It is a generalisation of the AHP decision methodology where hierarchies are replaced by networks, allowing the capturing of the outcome of dependence and feedback within and among the clusters of elements (Saaty, 2001). Its network structure differs from a hierarchy as illustrated in Figure 1. The hierarchy structure has a goal, levels of elements and connections between them. It has no inner dependence and no feedback from lower to higher levels. Unlike the hierarchy, the network has no levels but clusters of elements where every element may depend on any other element.

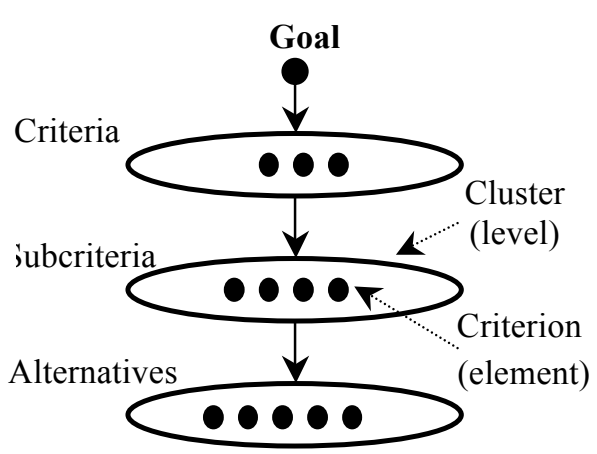

(a)

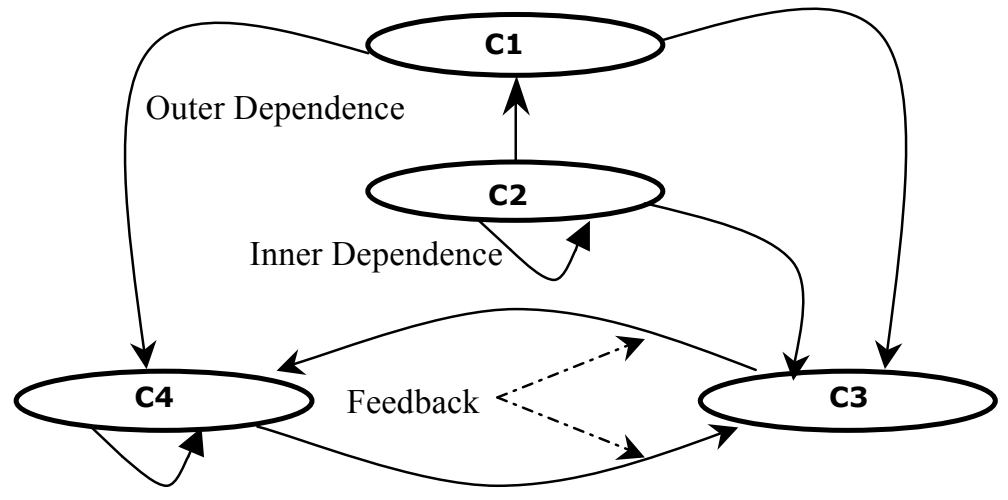

(b)

Figure 1. A sample of a Hierarchy and a Network.

The influence is transmitted from one cluster to another (outer dependence) and back, either directly from the second cluster, or, by transiting through intermediate clusters along a path which sometimes can return to the original cluster forming a cycle (Saaty, 2005). The existence of feedback indicates there is mutual outer dependence of criteria in two different clusters, which prevents the problem from being modelled hierarchically due to the difficulty in deciding which cluster is higher/lower than the other. Also, because of inner dependence, the relationships between same level criteria are not represented hierarchically.

The ANP model is a coupling of two parts. The first part consists of a control network of criteria that controls the interactions in which the criteria should be identified, organized and prioritised in the framework of a control network. The second part is a network of influences among the factors and clusters, i.e. the influence of elements in the feedback system with respect to each of these criteria. Paired comparison judgments of homogeneous elements are performed and synthesised to obtain the priorities of these criteria. The ANP then joins all possible outcomes together in its structures and both judgement and logic are used to estimate the relative influence from which the overall answer is to be derived (Saaty, 2001).

\section{The proposed model}




\subsection{Setting selection criteria}

To adapt the ANP methodology for such a technology selection process, it is the foremost activity of the researcher to examine the relevant issues involved. Hence, the first task is the definition of the criteria that will be used for the choice of the appropriate technology for rural connectivity. The activities used to consolidate the final list of the selection criteria comprised of an intensive literature survey of past studies on similar problems, including: (Douligeris and Pereira, 1994), (Sasidhar and Min, 2005), (Chemane et al., 2005), (Andrew et al., 2005) and (Pipattanasomporn, 2004). In addition, further refinement of the initial criteria list was completed through the interactions with telecommunication experts both from industry and academia from all over the world, who were asked to provide feedback on potential selection criteria. A list of 31 selection criteria deemed to affect the planners' decision in the choice of rural telecommunications backbone infrastructure was finally consolidated as can be observed in Table 1.

In order to rank the selection criteria according to their relative importance, an online questionnaire was designed. Telecommunication experts were asked to rate the importance of each criterion using a five-point Likert-type scale. The results were then analysed using SPSS (SPSS Inc., 2006), and univariate descriptive statistics were generated, including the relative importance index for each criterion. Table 1 summarises the obtained results, in which the mean value of each criterion is used for importance ordering. Moreover, all criteria have been grouped into six clusters, coded A through F. Each cluster only includes criteria that are comparable or do not differ by orders of magnitude (Saaty, 2005). Table 1 shows the coding and the ordering of criteria for all clusters.

Table 1. Ordering and clustering of criteria according to relevance and importance.

\begin{tabular}{|c|c|c|}
\hline Cluster & Criteria & Mean \\
\hline \multirow{9}{*}{ (A) Technical } & (A1) Reliability & 4.00 \\
\hline & (A2) Ease of maintenance & 3.94 \\
\hline & (A3) Remote network management & 3.88 \\
\hline & (A4) Compatibility & 3.81 \\
\hline & (A5) Ease of installation & 3.72 \\
\hline & (A6) Scalability & 3.54 \\
\hline & (A7) Bandwidth & 3.53 \\
\hline & (A8) Flexibility & 3.52 \\
\hline & (A9) Latency & 3.30 \\
\hline \multirow{8}{*}{ (B) Infrastructure } & (B1) Coverage range & 3.80 \\
\hline & (B2) Security of physical infrastructure & 3.73 \\
\hline & (B3) Proposed usage & 3.40 \\
\hline & (B4) Availability of skilled technicians & 3.34 \\
\hline & (B5) Access to existing telecoms infrastructure & 3.32 \\
\hline & (B6) Remoteness of area & 3.26 \\
\hline & (B7) Rollout time & 3.11 \\
\hline & (B8) Parallel infrastructure & 2.97 \\
\hline \multirow{5}{*}{ (C) Economical } & (C1) Operating cost & 4.13 \\
\hline & (C2) Funding sources & 4.11 \\
\hline & (C3) Capital cost & 3.98 \\
\hline & (C4) Return on investment & 3.63 \\
\hline & (C5) Economic development of area & 3.32 \\
\hline \multirow{4}{*}{ (D) Social } & (D1) Demand & 3.77 \\
\hline & (D2) Affordability & 3.73 \\
\hline & (D3) Population density & 3.48 \\
\hline & (D4) Community of interest & 3.42 \\
\hline \multirow{3}{*}{ (E) Regulatory } & (E1) Spectrum availability & 3.74 \\
\hline & (E2) Licensing constraints & 3.52 \\
\hline & (E3) Rights of way & 3.30 \\
\hline \multirow{2}{*}{ (F) Environmental } & (F1) Terrain topography & 3.24 \\
\hline & (F2) Climatic conditions & 3.00 \\
\hline
\end{tabular}




\subsection{Identification of potential alternatives for the model}

In order to identify potential technology alternatives, the reviewed published literature e.g. (Kawasumi, 2007), identified four technological solutions to provide rural backbone infrastructure to promote e-services in rural areas of developing countries. They include two wireline technologies: Fiber Optic Cable and Power Line Communication, and two wireless technologies: Fixed Wireless and Satellite, which were initially highlighted as candidate decision alternatives for this research. After consultation with telecommunication experts, the alternatives finally selected for this model are Fiber Optic Cable (G1), Power Line Communication (G2), Microwave Links (G3) and Satellite Communications (G4). Table 2 briefly summarises some characteristics of such infrastructure technologies.

Table 2. Comparisons of some features of potential alternatives.

\begin{tabular}{|c|c|c|}
\hline Alternative & Advantages & Disadvantages \\
\hline (G1) Fiber Optic Cable & $\begin{array}{l}\text { High speed } \\
\text { More reliability } \\
\text { High flexibility }\end{array}$ & $\begin{array}{l}\text { High Cost } \\
\text { Long rollout time } \\
\text { Most difficult to deploy }\end{array}$ \\
\hline (G2) Power Line Communication & \begin{tabular}{|l|} 
Simplicity \\
Low cost \\
Use of power lines \\
\end{tabular} & $\begin{array}{l}\text { Less reliability } \\
\text { Data signal disruption } \\
\text { Noise and interference } \\
\end{array}$ \\
\hline (G3) Microwave Link & $\begin{array}{l}\text { High speed } \\
\text { Low cost equipment } \\
\text { Fast deployment }\end{array}$ & $\begin{array}{l}\text { Low reach \& Line of sight } \\
\text { Licensing constraints } \\
\text { Less bandwidth \& Flexibility }\end{array}$ \\
\hline (G4) Satellite Communication & $\begin{array}{l}\text { Wide coverage } \\
\text { Ease of deployment } \\
\text { Overcomes topography }\end{array}$ & $\begin{array}{l}\text { High latency } \\
\text { High cost } \\
\text { Limited bandwidth }\end{array}$ \\
\hline
\end{tabular}

\subsection{Assessing dependency among criteria}

In order to examine the influence among criteria, a new survey questionnaire was distributed to experts, who were asked to assess the strength of dependency among criteria. Seven completed questionnaires were collected. The majority rule was then used to aggregate the responses into a single matrix, where a majority condition of 4 out of $7(4 / 7)$ experts' consensus (i.e. 57\%) was considered as a minimum requirement for any entry to confirm the existence of a direct relationship between any pair of criteria. The final dependency matrix was developed using a zero-one matrix of criteria against criteria using a binary value of 1 to signify dependence of one criterion on another, and zero otherwise (Saaty, 2006). Due to space limitation, such a matrix can not be presented in this paper. However, the authors may provide it upon request.

Figure 2 shows the network model which was constructed using the design module of the SuperDecisions software (Saaty, 2003). The said figure illustrates the entire inner dependence among elements within each cluster, i.e. the parent element and the elements to be compared are in the same cluster so that the cluster is linked to itself and a loop link appears. It indicates that the connections between the elements are in the same cluster. For example, element A8 means A2, A4, A5, A6 are interrelated with respect to A8. The environmental and alternative clusters have no inner dependence. Figure 2 also shows outer dependence which is the relationship between elements in one cluster with other elements in other clusters. For example, when considering A8, the elements G1, G2 G3 and G4 in G 'the alternative cluster' are interconnected and pairwise compared with respect to A8 in the 'technical' cluster A. The exception is the regulatory and environmental clusters, i.e. none of the elements in both clusters depend on elements from other clusters with respect to a common attribute within a cluster. Also, the technical and social clusters have no outer dependence on the economical cluster. Feedback links in which one compares the alternatives with respect to criteria, as in a hierarchy, and also compares the dominance of one criterion versus another for each alternative exist in this structure. For instance, in addition to separately comparing G1, G2, G3 and G4 with respect to $\mathrm{A} 1$ and $\mathrm{A} 7, \mathrm{~A} 1$ and $\mathrm{A} 7$ must also be compared with respect to G1. A pairwise question to be asked is: what is a more dominant characteristic of fiber optic cable technology, its reliability or its bandwidth? However, since feedback involves cycles, and cycling can be an infinite process, the operations needed to derive the priorities become more demanding than with hierarchies (Saaty, 2006). 


\section{Y. Gasiea, M. Emsley \& L. Mikhailov}

Based on the above, the developed inner and outer dependence and feedback among the network structure shown in Figure 2 excludes the hierarchy form and calls for the network form to model the selection of rural telecommunications infrastructure technology.

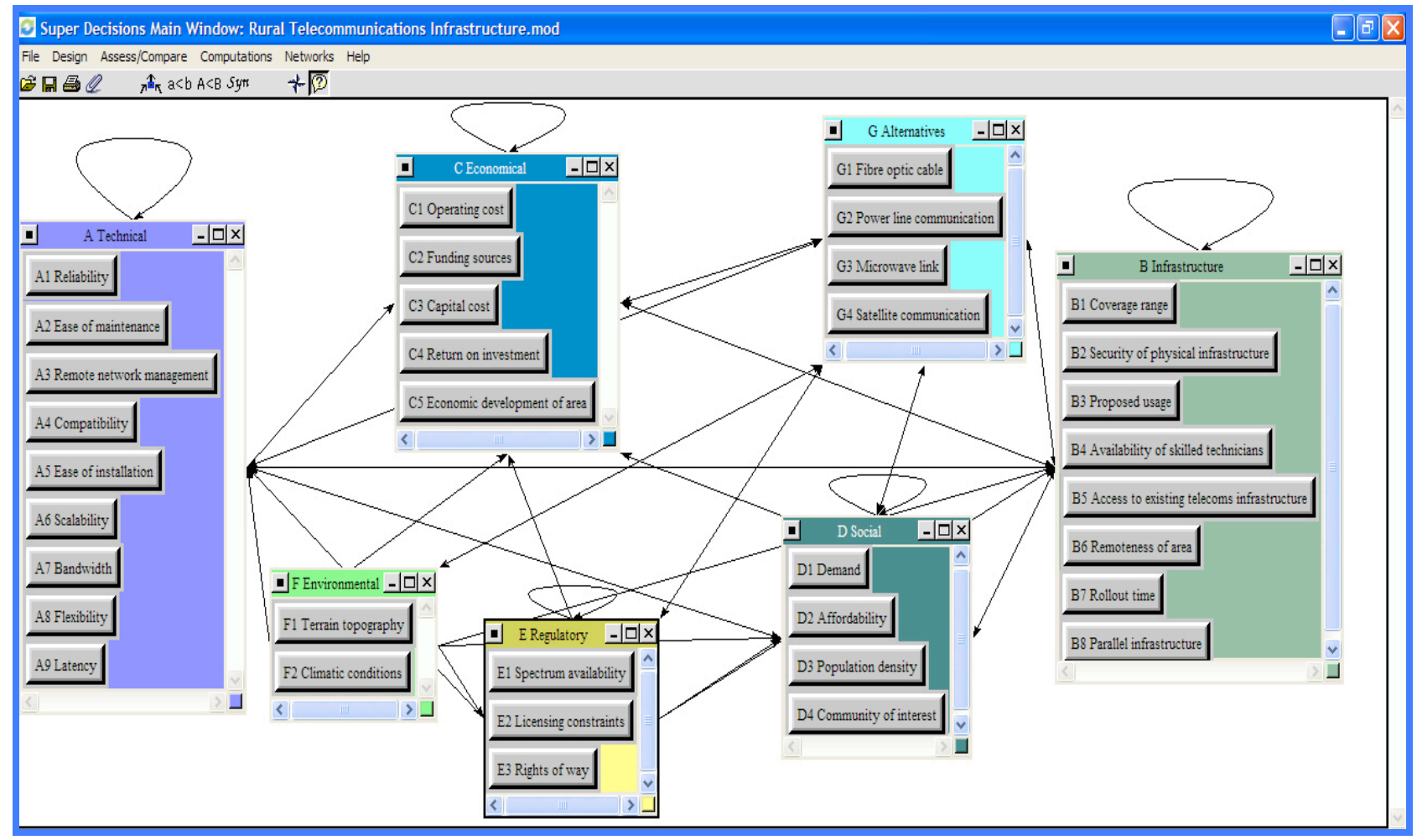

Figure 2. The proposed ANP network model with connections among elements/clusters.

\subsection{Pairwise comparisons}

After constructing the ANP model, the next phase is the measurement and data collection stage, which involves obtaining judgements from experts for pairwise comparison questions created according to the structure developed in the developed dependency matrix. This process requires a series of pairwise comparisons where the decision maker will compare two components at a time with respect to a control/parent criterion based on the nine-point scale suggested by Saaty (1988). A score of 1 indicates the equality between the two elements whereas score 9 represents the dominance of row element in the matrix over the column element. A reciprocal value is automatically assigned in the opposite position in the matrix.

In order to obtain the corresponding pairwise judgment matrices relevant pairwise comparisons matrices are constructed according to the dependency matrix described above. In this model, there are 92 judgement matrices which include 674 pairwise comparison questions. It is obvious that the task of asking such a large number of questions would be very enormous and would require intensive efforts and extended time. Hence, in order to economise efforts, it was decided to design and use several online questionnaires to gather data from experts. The questionnaires included all required pairwise questions to assess expert judgments in relation to the relative influence of affecting elements on the affected ones. An example of such posed pairwise questions is of the following kind: In selecting an appropriate backbone infrastructure technology in rural areas of developing countries, which influences Fiber optic cable technology more, ease of installation or ease of maintenance? Conversely, given ease of installation, which of these technologies are more dominant, Fiber optic cable or Satellite? Once the pairwise comparisons are completed, the authors computed the geometric means in order to aggregate individual judgments into a representative group judgment for each pairwise question. The Assess/Compare command of the SuperDecisions was then used to enter the obtained group judgments and initiating the comparison process. An example of the used comparison questionnaire mode is shown in Figure 3. 


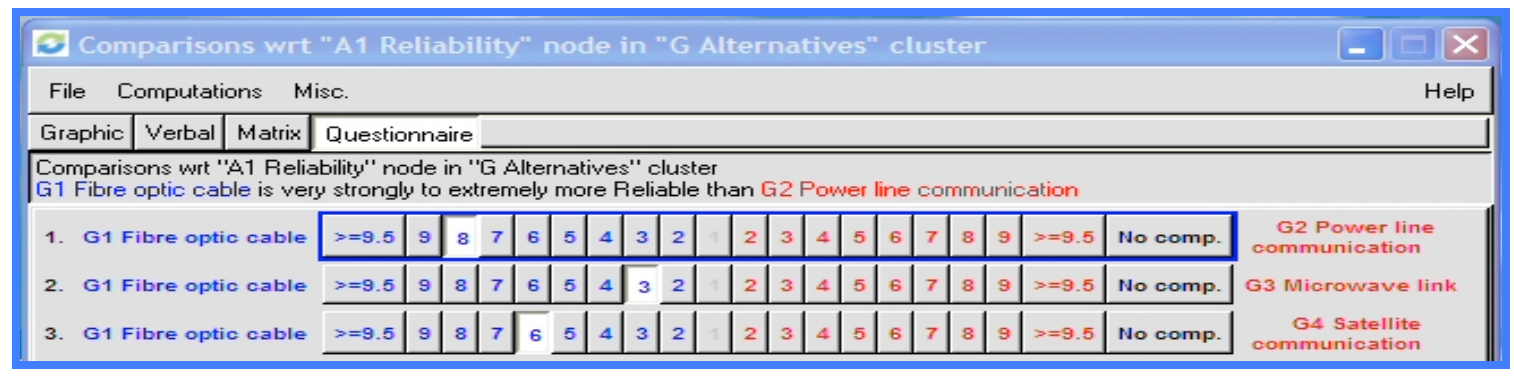

Figure 3. A partial view of SuperDecisions pairwise comparison questionnaire.

\subsection{Results and discussion}

The next stage of the process includes the computations of the relative importance of the elements. The SuperDecisions computations command has been used to perform necessary matrix algebra in relation to the creation of the three supermatrices associated with this model. From the obtained limit matrix, one can simply read the global priorities of the criteria and the alternatives from any column. Figure 4 presents the final results 'global preferences' for alternatives which were obtained using SuperDecisions 'Synthesis' command. These results indicate that the Microwave link technology is the most preferred alternative with a priority of $45.32 \%$, Satellite communication has $28.72 \%$, Fiber optic cable has $14.38 \%$ and the least preferred technology among others is Power line communication with a priority of $11.58 \%$. According to the 'Ideals' priorities; Microwave has a priority of 1.0, i.e. $100 \%$, so Satellite, Fiber optic and Power line are $63.37 \%, 31.74 \%$ and $25.55 \%$, respectively, as suitable backbone infrastructure technologies as Microwave.

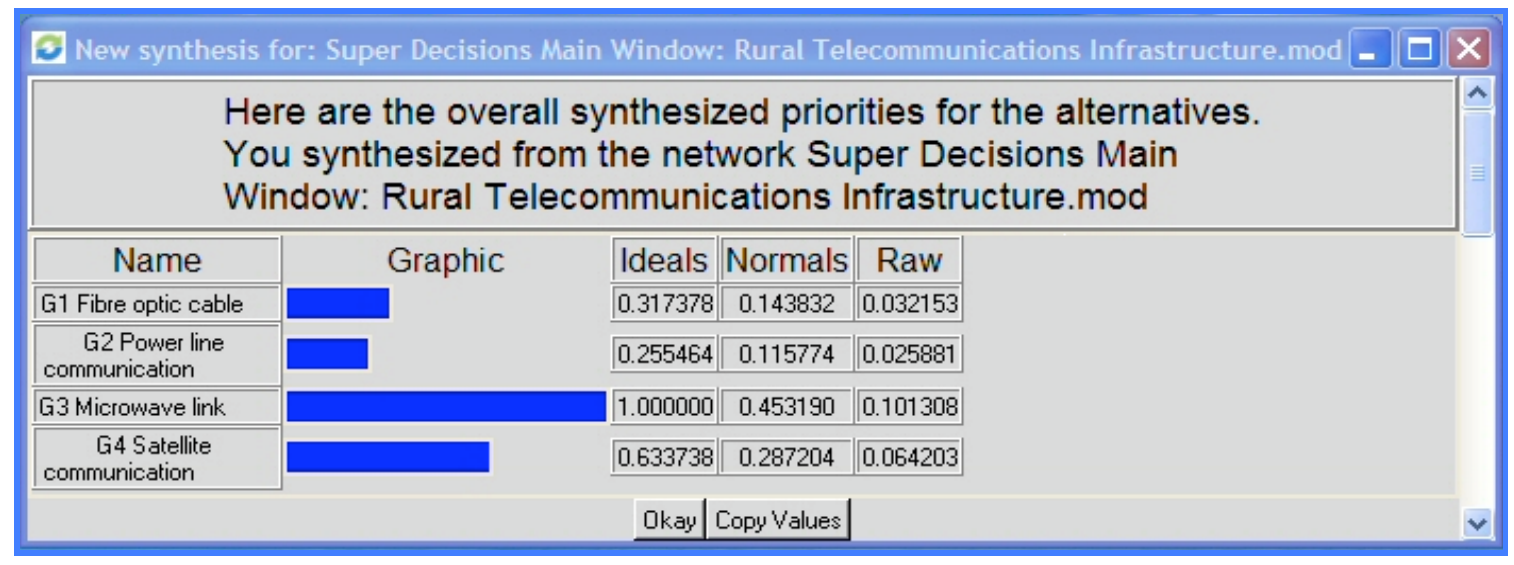

Figure 4. Final results as obtained from SuperDecisions Synthesis command.

\section{Conclusion}

This research paper reports on the applicability of using a MCDM method to enhance the selection process of an essential rural infrastructure technology. The above analysis indicates that the developed inner and outer dependence and feedback among the network structure shown in Figure 2 excludes the hierarchy form and calls for the network form to model the selection process. Owing to the time consuming nature of the ANP approach, its applications are noticeably less than that of the AHP. However, with the availability of an experienced focus group that can provide reliable judgements, the time taken for the entire process can be minimised and telecommunications planners can reach proper decisions concerning the selection. This is only an exercise to illustrate use of the ANP method and no real life conclusions should be drawn from it as each telecommunication infrastructure provider will have its own set of criteria. The intention here is to present a generic model based on factors and alternatives identified from the published literature, best practices and telecommunications experts that could then be adapted or extended to support a particular context or a situation of a developing country. The obtained final results reflect the preferences of experts who made the judgments, integrating their knowledge and experience and, therefore, not to be considered an objective assessment of the relative suitability of the four technologies as backbone infrastructure in rural areas. Final alternatives scores should, therefore, be thought of as an input to the decision-making process rather than its end. This process would be refined with experience, optimising the accuracy and time taken to reach proper decisions regarding the choice of telecommunication infrastructure in rural surroundings. 


\section{Y. Gasiea, M. Emsley \& L. Mikhailov}

\section{REFERENCES}

Andrew, T. N., Rahoo, P. \& Nepal, T. (2005). Enhancing the selection of communication technology for rural telecommunications: An analytic hierarchy process model. International Journal of Computers, Systems and Signals, 6 (2).

Chemane, L., Ekenberg, L., Popov, O. \& Cossa, T. (2005). MCDM model for selecting internet access technologies - A case study in Mozambique. IEEE EUROCON 2005.

Douligeris, C. \& Pereira, J. (1994). Telecommunications quality study using the analytic hierarchy process. IEEE journal on selected areas in communications 12 (2), 241-250

Kawasumi, Y., Miyoshi, Y. \& Koizumi, K. (2007). How to provide broadband access for rural and remote areas of developing countries. PTC'07: Telecom with vision. Honolulu, Hawaii.

Meade, L. M. \& Presley, A. (2002). R\&D project selection using the analytic network process. IEEE transactions on engineering management, 49, 59-66.

Nazem, S. M., Shi, Y., Lee, H., Sung Yeol, K., Tae Ho, P. \& Myung Ho, S. (2001). Multicriteria hub decision making for rural area telecommunication networks. European journal of operational research, 133 (3), 483-495.

Nepal, T. (2005). Evaluation of rural telecommunications infrastructure in South Africa. Problems of nonlinear analysis in engineering systems (Kazan), 3 (24), 138-149.

Pipattanasomporn, M. (2004). A study of remote area internet access with embedded power generation. Doctoral thesis. Alexandria, University of Virginia.

Saaty, T. L. (1996 ). Decision making with dependence and feedback: The analytic network process. Pittsburgh, PA: RWS Publications.

Saaty, T. L. (2001). Decision making with dependence and feedback, the analytic network process. Pittsburgh, PA: RWS Publications.

SAATY, R. W. (2003). Decision making in complex environments: A tutorial for the SuperDecisions software. Creative decisions foundation.

Saaty, T. L. (2005). Theory and applications of the analytic network process: decision making with benefits, opportunities, costs, and risks. Pittsburgh, PA: RWS Publications.

Saaty, T. L. (2006). Decision making with the analytic network process: Economic, political, social and technological applications with benefits, opportunities, costs and risks, Springer; 1st edition.

Sasidhar, M. \& Min, K. J. (2005). Decision support models for the selection of internet access technologies in rural communities. Telemat. Inf., 22 (3), 201-219.

Shang, J. S., Tjader, Y. \& Ding, Y. (2004). A unified framework for multicriteria evaluation of transportation projects. IEEE transactions on engineering management, 51(3). 